\title{
A model for a large investor trading at market indifference prices. I: single-period case.
}

\author{
P. Bank * \\ Technische Universität Berlin \\ and Quantitative Products Laboratory \\ Institut für Mathematik \\ Straße des 17. Juni 135, 10623 Berlin, Germany \\ (bank@math.tu-berlin.de) \\ D. Kramkov ${ }^{\dagger}$ \\ Carnegie Mellon University, \\ Department of Mathematical Sciences, \\ 5000 Forbes Avenue, Pittsburgh, PA, 15213-3890, US \\ (kramkov@cmu.edu)
}

October 8, 2018

\begin{abstract}
We develop a single-period model for a large economic agent who trades with market makers at their utility indifference prices. We compute the sensitivities of these market indifference prices with respect to the size of the investor's order. It turns out that the price impact of an order is determined both by the market makers' joint risk tolerance and by the variation of individual risk tolerances. On a technical

*This research was supported in part by the National Science Foundation under grant

${ }^{\dagger}$ The author also holds a part-time position at the University of Oxford. This research was supported in part by the National Science Foundation under grant DMS-0505414 and by the Oxford-Man Institute for Quantitative Finance at the University of Oxford.
\end{abstract} DMS-0505021. 
level, a key role in our analysis is played by a pair of conjugate saddle functions associated with the description of Pareto optimal allocations in terms of the aggregate utility function.

Keywords: aggregate utility function, Bertrand competition, demand pressure, equilibrium, large investor, liquidity, Pareto allocation, price impact, risk-tolerance, utility indifference prices.

JEL Classification: G11, G12, G13, C61.

AMS Subject Classification (2010): 52A41, 60G60, 91G10, 91G20.

\section{Introduction}

Most models studied in Mathematical Finance specify price dynamics exogenously, e.g., by some semi-martingale which satisfies certain economically sensible conditions, most notably the absence of arbitrage opportunities, and whose behavior is not affected by an investor's trading strategy. While being linear with respect to the order flow and therefore convenient to analyze, such models inevitably ignore the fundamental economic principle that prices are formed by demand and supply. This idealization is justified from a practical viewpoint as long as the trading volume remains small enough to be easily covered by market liquidity. But when transactions involve a sizable part of 'the market' for a security, a model for their impact on asset prices is clearly called for.

It is the purpose of this paper and its companion [5] to systematically develop such a price impact model in, respectively, a one-period and a continuoustime framework. Of course, price impact models have been proposed before with different goals in mind and we refer to the survey by Gökay et al. [18] for a more detailed overview. For instance, Almgren and Chriss [1] and Schied and Schöneborn [30] seek to find optimal execution strategies for selling a large quantity of shares, while Frey and Stremme [15], Platen and Schweizer [27], Papanicolaou and Sircar [26] and Cvitanić and Ma [11] as well as Çetin et al. [10] focus on option pricing in illiquid markets. The impact of transactions on asset prices in their models is typically specified explicitly, in a mathematically convenient form, but not derived systematically from equilibrium-based considerations of economic theory. 
There is a vast literature on price formation in economics and we refer to Amihud et al. [2], Biais et al. [9] and O'Hara [25] for surveys of financial models with price impact. As in the model of Stoll [31], also in our framework prices are quoted by some market makers and the price impact is due to their risk aversion against growing inventories of risky securities. In line with the utility based valuation methods common in mathematical finance, we add the feature that our market makers can hedge against the incurred risk by trading freely among themselves. We argue that, due to competition, this hedging opportunity leads to more favorable quotes for the proposed transaction and in fact eventually leaves every market maker indifferent between the pre- and the post-transaction allocation of wealth.

We develop a mathematical framework for the analysis of these market indifference prices. The key tool in our study is the convex duality theory for saddle functions as presented by Rockafellar [29]. This allows us, for instance, to compute the asymptotic expansion of market indifference prices with respect to the order size. It turns out that these expansions have a component which can be described in terms of a representative agent's risk aversion and another component which reflects the diversity of risk aversions among the market makers.

The paper is structured as follows. In Section 2 we give the mathematical specification of our model and continue the discussion of its economic features and some of the pertaining literature. In this section we also obtain a basic result on the existence and uniqueness of market indifference prices. A detailed study of these prices including the computation of their sensitivities with respect the order size constitutes our main result and is presented in Section 4. This analysis relies on a number of technical facts related to the classical parametrization of Pareto optimal allocations in terms of the aggregate utility function. They are collected in Section 3.

\section{Model}

We consider a single-period financial model with initial time 0 and maturity 1 where $M \in\{1,2, \ldots\}$ market makers quote prices for $J \in\{1,2, \ldots\}$ traded assets. These assets are specified through their payoffs $\psi=\left(\psi^{1}, \ldots, \psi^{J}\right)$ at time 1 which are random variables defined on a complete probability space $(\Omega, \mathscr{F}, \mathbb{P})$. As usual, we identify random variables differing on a set of measure zero and use the notations $\mathbf{L}^{0}\left(\mathbf{R}^{d}\right)$, for the metric space of such equiva- 
lence classes with values in $\mathbf{R}^{d}$ and convergence in probability, and $\mathbf{L}^{p}\left(\mathbf{R}^{d}\right)$, $p \geq 1$, for the Banach space of $p$-integrable random variables.

The way the market makers serve the incoming orders crucially depends on their attitude toward risk, which we model in the classical framework of expected utility. Thus, we interpret the probability measure $\mathbb{P}$ as a description of the common beliefs of our market makers (same for all, for simplicity) and denote by $u_{m}=\left(u_{m}(x)\right)_{x \in \mathbf{R}}$ market maker $m$ 's utility function for terminal wealth.

Assumption 2.1. Each $u_{m}=u_{m}(x), m=1, \ldots, M$, is a strictly concave, strictly increasing, continuously differentiable, and bounded above function on the real line $\mathbf{R}$ satisfying

$$
\lim _{x \rightarrow \infty} u_{m}(x)=0
$$

The normalization to zero in (2.1) is added only for notational convenience. Many of our results will be derived under the following additional condition on the utility functions, which, in particular, implies their boundedness from above.

Assumption 2.2. Each utility function $u_{m}=u_{m}(x), m=1, \ldots, M$, is twice continuously differentiable and its absolute risk aversion coefficient is bounded away from zero and infinity, that is, for some $c>0$,

$$
\frac{1}{c} \leq a_{m}(x) \triangleq-\frac{u_{m}^{\prime \prime}(x)}{u_{m}^{\prime}(x)} \leq c, \quad x \in \mathbf{R} .
$$

The prices quoted by the market makers are also influenced by their initial endowments $\alpha_{0}=\left(\alpha_{0}^{m}\right)_{m=1, \ldots, M} \in \mathbf{L}^{0}\left(\mathbf{R}^{M}\right)$, where $\alpha_{0}^{m}$ is an $\mathscr{F}$-measurable random variable describing the terminal wealth of the $m$ th market maker if no orders have to be filled. We assume that the initial allocation $\alpha_{0}$ is Pareto optimal, that is, there is no strictly better re-allocation of the same resources in the sense of the following well-known

Definition 2.3. A vector of $\mathscr{F}$-measurable random variables $\alpha=\left(\alpha^{m}\right)_{m=1, \ldots, M}$ is called a Pareto optimal allocation if

$$
\mathbb{E}\left[\left|u_{m}\left(\alpha^{m}\right)\right|\right]<\infty, \quad m=1, \ldots, M,
$$


and if there is no other allocation $\beta \in \mathbf{L}^{0}\left(\mathbf{R}^{M}\right)$ with the same total endowment,

$$
\sum_{m=1}^{M} \beta^{m}=\sum_{m=1}^{M} \alpha^{m}
$$

which leaves all market makers not worse and at least one of them better off in the sense that

$$
\mathbb{E}\left[u_{m}\left(\beta^{m}\right)\right] \geq \mathbb{E}\left[u_{m}\left(\alpha^{m}\right)\right] \text { for all } m=1, \ldots, M
$$

and

$$
\mathbb{E}\left[u_{m}\left(\beta^{m}\right)\right]>\mathbb{E}\left[u_{m}\left(\alpha^{m}\right)\right] \text { for some } m \in\{1, \ldots, M\}
$$

Suppose that at time 0 the market makers fill an order from a large investor for the quantity $q \in \mathbf{R}^{J}$ of the assets $\psi \in \mathbf{L}^{0}\left(\mathbf{R}^{J}\right)$. As a result, the market makers' total endowment changes from $\Sigma_{0} \triangleq \sum_{m=1}^{M} \alpha_{0}^{m}$ to

$$
\Sigma(x, q) \triangleq \Sigma_{0}+x+\langle q, \psi\rangle=\Sigma_{0}+x+\sum_{j=1}^{J} q^{j} \psi^{j}
$$

where $\langle\cdot, \cdot\rangle$ denotes the Euclidean scalar product and $x \in \mathbf{R}$ and $q \in \mathbf{R}^{J}$ stand for, respectively, the cash amount and the number of assets acquired by the market makers. Our model will assume that $\Sigma(x, q)$ is re-allocated among the market makers in the form of a Pareto optimal allocation. For this to be possible we have to impose

Assumption 2.4. For any $x \in \mathbf{R}$ and $q \in \mathbf{R}^{J}$ there is an allocation $\beta \in$ $\mathbf{L}^{0}\left(\mathbf{R}^{M}\right)$ with the total endowment $\Sigma(x, q)$ such that

$$
\mathbb{E}\left[u_{m}\left(\beta^{m}\right)\right]>-\infty, \quad m=1, \ldots, M
$$

Remark 2.5. Under Assumption 2.1, Lemma 3.2 below shows that Assumption 2.4 is equivalent to the finiteness of the aggregate expected utility. Under Assumptions 2.1 and 2.2, Lemma 3.4 proves that Assumption 2.4 is also equivalent to the existence of all exponential moments for $\psi$ under the pricing measure $\mathbb{Q}_{0}$ associated with the initial Pareto optimal allocation $\alpha_{0}$. 


\subsection{Market indifference prices}

Theorem 2.6 below identifies uniquely both the transaction price $x=x(q)$ associated with an order $q$ and the market makers' post-transaction allocation of wealth $\alpha_{1}=\alpha_{1}(q)$ under the following two conditions:

Pareto optimality: The random endowment $\Sigma(x, q)$ is redistributed between the market makers to form a new Pareto optimal allocation $\alpha_{1}$.

Utility indifference: The market makers' expected utilities do not change:

$$
\mathbb{E}\left[u_{m}\left(\alpha_{1}^{m}\right)\right]=\mathbb{E}\left[u_{m}\left(\alpha_{0}^{m}\right)\right], \quad m=1, \ldots, M .
$$

We postpone the discussion of the economic motivation behind these two conditions until Section 2.2. By analogy with the popular utility-based valuation method in mathematical finance, we call $x=x(q)$ the market indifference price of the order $q$.

Theorem 2.6. Under Assumptions 2.1 and 2.4, every position $q \in \mathbf{R}^{J}$ yields a unique cash amount $x=x(q)$ and a unique Pareto optimal allocation $\alpha_{1}=$ $\alpha_{1}(q)$ of $\Sigma(x, q)$ preserving the market makers' expected utilities in the sense of (2.5).

Proof. For a real number $y$ denote by $\mathscr{B}(y)$ the family of allocations $\beta=$ $\left(\beta^{m}\right)_{m=1, \ldots, M}$ with total endowment less than $\Sigma(y, q)$ and such that

$$
\mathbb{E}\left[u_{m}\left(\beta^{m}\right)\right] \geq \mathbb{E}\left[u_{m}\left(\alpha_{0}^{m}\right)\right], \quad m=1, \ldots, M .
$$

By Assumptions 2.1 and 2.4, this set is non-empty for sufficiently large $y$ and, by the concavity of utility functions, is a convex subset of $\mathbf{L}^{0}\left(\mathbf{R}^{M}\right)$. Denote

$$
\widehat{y} \triangleq \inf \{y \in \mathbf{R}: \mathscr{B}(y) \neq \emptyset\},
$$

let $\left(y_{n}\right)_{n \geq 1}$ be a strictly decreasing sequence of real numbers converging to $\widehat{y}$, and arbitrarily choose $\beta_{n} \in \mathscr{B}\left(y_{n}\right), n \geq 1$.

From Assumption 2.1 we deduce the existence of $c>0$ such that, for $m=1, \ldots, M$,

$$
y^{-} \leq c\left(-u_{m}(y)\right), \quad y \in \mathbf{R},
$$

where $y^{-} \triangleq \max (0,-y)$; for example, we can take

$$
c=1 / \min _{m=1, \ldots, M} u_{m}^{\prime}(0) .
$$


It follows that

$$
\left.\mathbb{E}\left[\left(\beta_{n}^{m}\right)^{-}\right)\right] \leq c \mathbb{E}\left[\left(-u_{m}\left(\beta_{n}^{m}\right)\right)\right] \leq c \mathbb{E}\left[\left(-u_{m}\left(\alpha_{0}^{m}\right)\right)\right]<\infty, \quad n \geq 1,
$$

and, therefore, the sequence $\left(\left(\beta_{n}\right)^{-}\right)_{n \geq 1}$ is bounded in $\mathbf{L}^{1}\left(\mathbf{R}^{M}\right)$. Since, in addition,

$$
\sum_{m=1}^{M} \beta_{n}^{m} \leq \Sigma\left(y_{n}, q\right) \leq \Sigma\left(y_{1}, q\right),
$$

the family of all possible convex combinations of $\left(\beta_{n}\right)_{n \geq 1}$ is bounded in $\mathbf{L}^{0}\left(\mathbf{R}^{M}\right)$.

By Lemma A1.1 in [13] we can then choose convex combinations $\zeta_{n}$ of $\left(\beta_{k}\right)_{k \geq n}, n \geq 1$, which converge almost surely to a random variable $\zeta \in$ $\mathbf{L}^{0}\left(\mathbf{R}^{M}\right)$. Clearly,

$$
\sum_{m=1}^{M} \zeta^{m} \leq \Sigma(\widehat{y}, q)
$$

Since the utility functions are bounded from above, Fatou's lemma yields

$$
\mathbb{E}\left[u_{m}\left(\zeta^{m}\right)\right] \geq \limsup _{n \rightarrow \infty} \mathbb{E}\left[u_{m}\left(\zeta_{n}^{m}\right)\right] \geq \mathbb{E}\left[u_{m}\left(\alpha_{0}^{m}\right)\right]
$$

where the second estimate holds because $\zeta_{n} \in \mathscr{B}\left(y_{n}\right)$ by the convexity of $\mathscr{B}\left(y_{n}\right)$. It follows that $\zeta \in \mathscr{B}(\widehat{y})$. The minimality of $\widehat{y}$ then immediately implies the equalities in (2.6) and (2.7) and the Pareto optimality of $\zeta$. Hence, we can select $x=\widehat{y}$ and $\alpha_{1}=\zeta$, thus proving their existence.

Finally, the uniqueness of $x$ and $\alpha_{1}$ follows from the strict concavity of utility functions.

The preceding proof yields immediately that the market indifference price $x(q)$ is minimal in the following sense:

Corollary 2.7. Under Assumptions 2.1 and 2.4, the market indifference price $x(q)$ associated with an order $q \in \mathbf{R}^{J}$ is the minimal amount $x \in \mathbf{R}$ for which there exists an allocation $\beta \in \mathbf{L}^{0}\left(\mathbf{R}^{M}\right)$ with the total wealth $\Sigma(x, q)$ such that

$$
\mathbb{E}\left[u_{m}\left(\beta^{m}\right)\right] \geq \mathbb{E}\left[u_{m}\left(\alpha_{0}^{m}\right)\right], \quad m=1, \ldots, M .
$$

In Section 4 we show in detail how the market indifference price $x=$ $x(q): \mathbf{R}^{J} \rightarrow \mathbf{R}$ and the Pareto allocations $\alpha_{1}=\alpha_{1}(q): \mathbf{R}^{J} \rightarrow \mathbf{L}^{0}\left(\mathbf{R}^{M}\right)$ determined by Theorem 2.6 depend on the order $q$ of the large investor. 


\subsection{Economic considerations}

Let us next discuss the scope and limitations of our model as well as its relation to other models proposed in the literature. The book by O'Hara [25] as well as the surveys by Amihud et al. [2] and by Biais et al. [9] provide an extensive overview of the economic background.

Like in the work of Stoll [31] and Ho and Stoll [20] also in our model the market makers' quotes depend on the inventory they hold. The first distinctive feature of our setup is the assumption that the market makers share their risk in a Pareto optimal way. Since Pareto optimal allocations of wealth can be achieved, essentially, only in complete markets, we are implicitly postulating that, besides the publicly traded assets $\psi$, the market makers can also trade any arbitrarily structured product among themselves. In other words, they have a complete 'over the counter market' at their disposal. It would be interesting to obtain conditions under which this auxiliary market can be dispensed with and the desired completeness property is achieved endogenously by trading only the primary securities $\psi$. In this respect, we mention the works by Anderson and Raimondo [3], Hugonnier et al. [21], Riedel and Herzberg [28], Kramkov [22] on the existence of an endogenously complete Arrow-Radner equilibrium.

As a second key condition we assume that the market makers are indifferent between their pre- and post-transaction allocations of wealth. Given the existence of a complete market at their disposal, this can be viewed as a consequence of a Bertrand-style competition among them. Indeed, on one hand Corollary 2.7 shows that there is no way to trade $q$ securities at a transaction price less than $x(q)$ if no market maker is to loose in terms of expected utility. On the other hand, if one of the market makers could fill the order $q$ at a price $x>x(q)$ she could subsequently use a small part of the difference $x-x(q)$ to offer attractive deals to her fellow market makers on the over the counter market, e.g., by topping up the utility preserving allocation $\alpha_{1}(q)$ of Theorem 2.6. Plainly, all market makers would like to proceed this way. Following the usual Bertrand competition argument, this leads to a limiting quote of the market indifference price $x(q)$ and all the market makers end up being indifferent between the pre- and the post-transaction allocation of wealth. It may be interesting to note that the market indifference price $x(q)$ is smaller than every of the market makers' reservation prices $x_{m}(q)$ defined by

$$
\mathbb{E}\left[u_{m}\left(\alpha_{0}^{m}+x_{m}(q)+\langle q, \psi\rangle\right)\right]=\mathbb{E}\left[u_{m}\left(\alpha_{0}^{m}\right)\right], \quad m=1, \ldots, M
$$


This follows directly from Corollary 2.7 and is due to the market makers' ability to improve their positions by trading with each other.

There are, of course, several idealizations in our model. For instance, it assumes that all trades between the market markers are completed instantly after the transaction with the large investor. But in real markets it may take time to find counterparties for a hedge and it may be impossible to negotiate with them the best possible deals. Hence, to compensate for risks emerging from these frictions, real market makers will ask for a risk premium and for compensation of their operational costs both of which are not included in our transaction price; see Grossman and Miller [19], Duffie et al. [14], Longstaff [24] and the references therein. Another idealization is that the market makers do not act strategically. In particular, they do not account for adverse selection effects as studied, e.g., by Kyle [23], Back [4], Glosten and Milgrom [17], Biais et al. [8].

As pointed out by Bernhardt and Hughson [7] the utility indifference may not hold when, by contrast to our setting, orders can be split and market makers quote price schedules which have to be honored independently from the fellow market makers and when hedging opportunities are not taken into account. Our model is different from the one in Garleanu et al. [16] where a representative market maker maximizes utility from consumption and where prices are determined so that her optimal position matches some exogenously given demand for marketed assets. Our market makers also do not exert market power as investigated, e.g., in the work of Weretka [32]. While it would certainly be desirable to account for all these various aspects of market microstructure, this is clearly beyond the scope of a single paper.

\section{Parametrization of Pareto allocations}

The analysis of market indifference prices in Section 4 relies on the parametrization of Pareto allocations stated, in our setting, in Theorem 3.1 below. This parametrization is well-known; see, e.g., Dana and Le Van [12] for a similar result.

Recall that the aggregate utility function is defined as

$$
r(v, x) \triangleq \sup _{x^{1}+\cdots+x^{M}=x} \sum_{m=1}^{M} v^{m} u_{m}\left(x^{m}\right), \quad v \in(0, \infty)^{M}, x \in \mathbf{R} .
$$

One can show, see, e.g., Theorem 3.1 of [6], that under Assumption 2.1 
$r=r(v, x)$ is a continuously differentiable saddle function, strictly convex and decreasing in $v \in(0, \infty)^{M}$ and strictly concave and increasing in $x \in \mathbf{R}$. Moreover, for any $(v, x) \in(0, \infty)^{M} \times \mathbf{R}$, the supremum in (3.1) is attained at the vector $\widehat{x}=\left(\widehat{x}^{m}\right)_{m=1, \ldots, M} \in \mathbf{R}^{M}$ uniquely determined by

$$
v^{m} u_{m}^{\prime}\left(\widehat{x}^{m}\right)=\frac{\partial r}{\partial x}(v, x)
$$

or, equivalently, by

$$
u_{m}\left(\widehat{x}^{m}\right)=\frac{\partial r}{\partial v^{m}}(v, x), \quad m=1, \ldots, M .
$$

Theorem 3.1. Under Assumption 2.1, the following statements are equivalent for every $\Sigma \in \mathbf{L}^{0}(\mathbf{R})$ :

1. The allocation $\alpha=\left(\alpha^{m}\right)_{m=1, \ldots, M}$ of $\Sigma$ is Pareto optimal.

2. The random variables $\alpha=\left(\alpha^{m}\right)_{m=1, \ldots, M}$ satisfy the integrability condition (2.3) and there is a vector $v \in(0, \infty)^{M}$ such that

$$
v^{m} u_{m}^{\prime}\left(\alpha^{m}\right)=\frac{\partial r}{\partial x}(v, \Sigma), \quad m=1, \ldots, M
$$

Moreover, such a vector $v$ is defined uniquely up to a scalar multiple.

Proof. Denote by $\mathscr{B}=\mathscr{B}(\Sigma)$ the family of allocations $\beta \in \mathbf{L}^{0}\left(\mathbf{R}^{M}\right)$ with the total endowment $\sum_{m=1}^{M} \beta^{m}=\Sigma$ such that

$$
\mathbb{E}\left[u^{m}\left(\beta^{m}\right)\right]>-\infty, \quad m=1, \ldots, M .
$$

$1 \Longrightarrow 2$ : The integrability condition (2.3) for $\alpha$ holds by the definition of a Pareto optimal allocation. By the concavity of the utility functions, the set

$$
C \triangleq\left\{z \in \mathbf{R}^{M}: z^{m} \leq \mathbb{E}\left[u_{m}\left(\beta^{m}\right)\right], m=1, \ldots, M, \text { for some } \beta \in \mathscr{B}\right\}
$$

is convex and, by the Pareto optimality of $\alpha$, the point

$$
\widehat{z}^{m} \triangleq \mathbb{E}\left[u_{m}\left(\alpha^{m}\right)\right], \quad m=1 \ldots, M,
$$

belongs to the boundary of $C$. Hence, there is a non-zero $v \in \mathbf{R}^{M}$ such that

$$
\langle v, \widehat{z}\rangle \geq\langle v, z\rangle, \quad z \in C
$$


or, equivalently,

$$
\mathbb{E}\left[\sum_{m=1}^{M} v^{m} u_{m}\left(\alpha^{m}\right)\right]=\sup _{\beta \in \mathscr{B}} \mathbb{E}\left[\sum_{m=1}^{M} v^{m} u_{m}\left(\beta^{m}\right)\right]=\mathbb{E}[r(v, \Sigma)] .
$$

As $v \neq 0$, the properties of the utility functions in Assumption 2.1 imply that $v \in(0, \infty)^{M}$. By $(3.2)$, the fact that the upper bound above is attained at $\alpha$ is equivalent to (3.4).

$2 \Longrightarrow 1$ : By the definition of $r=r(v, x)$, for every $\beta \in \mathscr{B}$

$$
\sum_{m=1}^{M} v^{m} u_{m}\left(\beta^{m}\right) \leq r(v, \Sigma)=\sum_{m=1}^{M} v^{m} u_{m}\left(\alpha^{m}\right) .
$$

Given the integrability requirement (2.3), this clearly implies the Pareto optimality of $\alpha$.

Finally, we note that (3.4) determines $v$ uniquely up to a scalar multiple.

It is common to normalize the Pareto weight $v$ from Theorem 3.1 by restricting it to the set

$$
\mathbf{S}^{M} \triangleq\left\{w \in(0,1)^{M}: \sum_{m=1}^{M} w^{m}=1\right\},
$$

the interior of the simplex; such a $v \in \mathbf{S}^{M}$ is defined uniquely.

The following result allows us to parametrize the Pareto allocations in our economy by the set

$$
\mathbf{A} \triangleq(0, \infty)^{M} \times \mathbf{R} \times \mathbf{R}^{J}
$$

Lemma 3.2. Let Assumption 2.1 hold. Then Assumption 2.4 is equivalent to

$$
\mathbb{E}[r(v, \Sigma(x, q))]>-\infty, \quad(v, x, q) \in \mathbf{A} .
$$

In this case, for $a=(v, x, q) \in \mathbf{A}$, the random vector $\pi(a) \in \mathbf{L}^{0}\left(\mathbf{R}^{M}\right)$ defined by

$$
v^{m} u_{m}^{\prime}\left(\pi^{m}(a)\right)=\frac{\partial r}{\partial x}(v, \Sigma(x, q)), \quad m=1, \ldots, M,
$$

forms a Pareto optimal allocation. Conversely, for $(x, q) \in \mathbf{R} \times \mathbf{R}^{J}$, any Pareto allocation of the total endowment $\Sigma(x, q)$ is given by (3.7) for some $v \in(0, \infty)^{M}$. 
Proof. Under Assumption 2.4, there is an allocation $\beta=\left(\beta^{m}\right)_{m=1, \ldots, M}$ of $\Sigma(x, q)$ satisfying (2.4). Since,

$$
\sum_{m=1}^{M} v^{m} u_{m}\left(\beta^{m}\right) \leq r(v, \Sigma(x, q)) \leq 0,
$$

we obtain (3.6).

Assume now that (3.6) holds. By (3.2), the condition (3.7) is equivalent to

$$
\sum_{m=1}^{M} v^{m} u_{m}\left(\pi^{m}(a)\right)=r(v, \Sigma(x, q)) .
$$

As $v^{m}>0$ and $u_{m}<0, m=1, \ldots, M$, we deduce from (3.6) that $u_{m}\left(\pi^{m}(a)\right) \in$ $\mathbf{L}^{1}, m=1, \ldots, M$, which, in particular, yields Assumption 2.4. The Pareto optimality of $\pi(a)$ is now an immediate corollary of Theorem 3.1.

Finally, the fact that any Pareto allocation of $\Sigma(x, q)$ is given by (3.7), for some $v \in(0, \infty)^{M}$, follows from Theorem 3.1.

In view of the equivalence of (3.2) and (3.3), the Pareto allocation $\pi(a)$ can also be defined by

$$
u_{m}\left(\pi^{m}(a)\right)=\frac{\partial r}{\partial v^{m}}(v, \Sigma(x, q)), \quad m=1, \ldots, M .
$$

A well-known interpretation of the condition (3.7) for the Pareto allocation $\pi(a)$ is that our market makers agree on the valuation of marginal trades. The existence of the corresponding pricing measure $\mathbb{Q}(a)$ is established in the following lemma.

Lemma 3.3. Under Assumptions 2.1 and 2.4, for any $a=(v, x, q) \in \mathbf{A}$, there is a probability measure $\mathbb{Q}(a)$ such that

$$
\frac{d \mathbb{Q}(a)}{d \mathbb{P}}=\frac{\frac{\partial r}{\partial x}(v, \Sigma(x, q))}{\mathbb{E}\left[\frac{\partial r}{\partial x}(v, \Sigma(x, q))\right]}=\frac{u_{m}^{\prime}\left(\pi^{m}(a)\right)}{\mathbb{E}\left[u_{m}^{\prime}\left(\pi^{m}(a)\right)\right]}, \quad m=1, \ldots, M,
$$

where $\pi(a)=\left(\pi^{m}(a)\right)_{m=1, \ldots, M}$ is the Pareto optimal allocation defined in (3.7).

Proof. In view of Lemma 3.2 it is sufficient to verify that

$$
\mathbb{E}\left[\frac{\partial r}{\partial x}(v, \Sigma(x, q))\right]<\infty .
$$


This follows from (3.6) and the inequality

$$
\frac{\partial r}{\partial x}(v, \Sigma(x, q)) \leq r(v, \Sigma(x, q))-r(v, \Sigma(x-1, q)),
$$

which holds by the concavity of $r(v, \cdot)$.

We conclude this section with another equivalent re-formulation of Assumption 2.4, this time, in terms of an exponential moment condition for $\psi$ under the initial pricing measure $\mathbb{Q}_{0}$.

Lemma 3.4. Under Assumptions 2.1 and 2.2 the pricing measure $\mathbb{Q}_{0}$ of the initial Pareto allocation $\alpha_{0}=\left(\alpha_{0}^{m}\right)_{m=1, \ldots, M}$ is well-defined by

$$
\frac{d \mathbb{Q}_{0}}{d \mathbb{P}}=\frac{u_{m}^{\prime}\left(\alpha_{0}^{m}\right)}{\mathbb{E}\left[u_{m}^{\prime}\left(\alpha_{0}^{m}\right)\right]}, \quad m=1, \ldots, M
$$

and Assumption 2.4 is equivalent to the existence of all exponential moments of $\psi=\left(\psi^{j}\right)_{j=1, \ldots, J}$ under $\mathbb{Q}_{0}$ :

$$
\mathbb{E}_{\mathbb{Q}_{0}}\left[e^{z|\psi|}\right]<\infty, \quad z \in \mathbf{R} .
$$

Proof. Observe first that Assumptions 2.1 and 2.2 imply that

$$
\frac{1}{c} \leq-\frac{u_{m}^{\prime}(x)}{u_{m}(x)} \leq c, \quad x \in \mathbf{R}, \quad m=1, \ldots, M,
$$

with the same constant $c>0$ as in (2.2). From this and (3.2) we deduce that

$$
\frac{M}{c} \frac{\partial r}{\partial x}(v, x) \leq-r(v, x) \leq c M \frac{\partial r}{\partial x}(v, x), \quad(v, x) \in(0, \infty)^{M} \times \mathbf{R}
$$

and, therefore,

$$
\begin{aligned}
& -r(v, x+y) \geq \frac{M}{c} \frac{\partial r}{\partial x}(v, x) \exp \left(\frac{1}{c M} y^{-}-\frac{c}{M} y^{+}\right), \\
& -r(v, x+y) \leq c M \frac{\partial r}{\partial x}(v, x) \exp \left(\frac{c}{M} y^{-}-\frac{1}{c M} y^{+}\right),
\end{aligned}
$$

where $y^{+} \triangleq \max (y, 0)$ and $y^{-} \triangleq \max (-y, 0)$.

For $\mathbb{Q}_{0}$ to be well-defined we have to verify that

$$
\mathbb{E}\left[u_{m}^{\prime}\left(\alpha_{0}^{m}\right)\right]<\infty, \quad m=1, \ldots, M
$$


This follows from (3.11) and the inequality

$$
\mathbb{E}\left[u_{m}\left(\alpha_{0}^{m}\right)\right]>-\infty, \quad m=1, \ldots, M,
$$

which holds by the definition of a Pareto optimal allocation.

Let $v_{0} \in(0, \infty)^{M}$ denote a Pareto weight of $\alpha_{0}$ from Theorem 3.1. The positive homogeneity and the monotonicity properties of $r(\cdot, x)$ imply the equivalence of (3.6) and

$$
\mathbb{E}\left[r\left(v_{0}, \Sigma(x, q)\right)\right]>-\infty, \quad(x, q) \in \mathbf{R} \times \mathbf{R}^{J},
$$

which by (3.12) and the definition of $\mathbb{Q}_{0}$ is, in turn, equivalent to (3.10).

\section{Analysis of market indifference prices}

The construction of market indifference prices in Theorem 2.6 does not directly allow for an analysis of how these prices actually depend on the order the market makers' are assumed to fill. We thus develop in Section 4.1 below a quantitative description of these prices. Indeed, Theorem 4.1 shows in particular that the market indifference price and the associated Pareto allocation can be computed from the saddle conjugate of the representative market maker's expected utility. Theorem 4.2 then computes the second order derivatives of market indifference prices and the sensitivity of their Pareto allocations with respect to the order size. Section 4.2 then uses these to provide asymptotic expansions of market indifference prices which reveal their dependence on the market makers' individual and aggregate risk tolerances.

\subsection{Quantitative description of market indifference prices}

A key role in our study of market indifference prices will be played by the pair of conjugate saddle functions

$$
\begin{aligned}
& F_{0}(a) \triangleq \mathbb{E}[r(v, \Sigma(x, q))], \quad a=(v, x, q) \in \mathbf{A}, \\
& G_{0}(b) \triangleq \sup _{v \in(0, \infty)^{M}} \inf _{x \in \mathbf{R}}\left[\langle v, u\rangle+x y-F_{0}(v, x, q)\right], \quad b=(u, y, q) \in \mathbf{B},
\end{aligned}
$$

where $r=r(v, x)$, the aggregate utility function, and $\mathbf{A}$, the parameter set

of Pareto allocations, are defined in (3.1) and (3.5), and

$$
\mathbf{B} \triangleq(-\infty, 0)^{M} \times(0, \infty) \times \mathbf{R}^{J}
$$


These functions represent the initial values of the stochastic fields $F=$ $\left(F_{0}, F_{1}\right)$ and $G=\left(G_{0}, G_{1}\right)$; the terminal values are given by

$$
\begin{aligned}
& F_{1}(a) \triangleq r(v, \Sigma(x, q)), \quad a=(v, x, q) \in \mathbf{A}, \\
& G_{1}(b) \triangleq \sup _{v \in(0, \infty)^{M}} \inf _{x \in \mathbf{R}}\left[\langle v, u\rangle+x y-F_{1}(v, x, q)\right], \quad b=(u, y, q) \in \mathbf{B} .
\end{aligned}
$$

The stochastic fields $F$ and $G$ are thoroughly studied in our companion paper [6] under Assumption 2.1 and the condition:

$$
F_{0}(a)=\mathbb{E}[r(v, \Sigma(x, q))]>-\infty, \quad a=(v, x, q) \in \mathbf{A},
$$

which, by Lemma 3.2, is equivalent to Assumption 2.4.

Theorem 4.1 in [6] describes in detail the properties of the sample paths of the stochastic fields $F$ and $G$. In particular, the functions $F_{0}$ and $G_{0}$ are continuously differentiable, and the derivatives of $F_{0}$ can be computed by taking expectations of the corresponding derivatives of $F_{1}$ :

$$
\frac{\partial F_{0}}{\partial a^{i}}(a)=\mathbb{E}\left[\frac{\partial F_{1}}{\partial a^{i}}(a)\right], \quad a \in \mathbf{A} .
$$

Moreover, Theorem 2.2 in [6] shows that, for every fixed $q \in \mathbf{R}^{J}$, the following conjugacy relationships between $(v, x) \in(0, \infty)^{M} \times \mathbf{R}$ and $(u, y) \in$ $(-\infty, 0)^{M} \times(0, \infty)$ are equivalent:

1. We have $x=\frac{\partial G_{0}}{\partial y}(u, y, q)=G_{0}(u, 1, q)$ and $v=\frac{\partial G_{0}}{\partial u}(u, y, q)$.

2. We have $y=\frac{\partial F_{0}}{\partial x}(v, x, q)$ and $u=\frac{\partial F_{0}}{\partial v}(v, x, q)$.

In addition, in this case,

$$
\frac{\partial G_{0}}{\partial q}(u, y, q)=-\frac{\partial F_{0}}{\partial q}(v, x, q) .
$$

For $a \in \mathbf{A}$, recall the notations $\pi(a)$ and $\mathbb{Q}(a)$ for the Pareto allocation and the pricing measure from (3.7) and (3.9), respectively. Also recall that $\mathbf{S}^{M}$ denotes the interior of the simplex in $\mathbf{R}^{M}$. The following result improves Theorem 2.6.

Theorem 4.1. Under Assumptions 2.1 and 2.4, for every position $q \in \mathbf{R}^{J}$ there is a unique cash amount $x(q)$ and a unique Pareto optimal allocation $\alpha_{1}(q)$ with total endowment $\Sigma(x(q), q)$ such that

$$
U_{0}^{m} \triangleq \mathbb{E}\left[u_{m}\left(\alpha_{0}^{m}\right)\right]=\mathbb{E}\left[u_{m}\left(\alpha_{1}^{m}(q)\right)\right], \quad m=1, \ldots, M .
$$


The Pareto optimal allocation $\alpha_{1}(q)$ has the form

$$
\alpha_{1}(q)=\pi(a(q)), \quad a(q)=(w(q), x(q), q)
$$

where the weights $w(q) \in \mathbf{S}^{M}$ and the cash amount $x(q) \in \mathbf{R}$ are given by

$$
\begin{aligned}
w^{m}(q) & =\frac{\partial G_{0}}{\partial u^{m}}\left(U_{0}, 1, q\right) / \sum_{k=1}^{M} \frac{\partial G_{0}}{\partial u^{k}}\left(U_{0}, 1, q\right), \quad m=1, \ldots, M . \\
x(q) & =G_{0}\left(U_{0}, 1, q\right) .
\end{aligned}
$$

The function $x=x(q): \mathbf{R}^{J} \rightarrow \mathbf{R}$ is convex, continuously differentiable, and, for $q \in \mathbf{R}^{J}$,

$$
\frac{\partial x}{\partial q^{j}}(q)=-\mathbb{E}_{\mathbb{Q}(a(q))}\left[\psi^{j}\right], \quad j=1, \ldots, M
$$

Proof. The uniqueness of the cash amount and of the Pareto optimal allocation with the desired properties follows directly from the definition of Pareto optimality and the strict concavity of utility functions.

For the existence, consider the cash amount $x(q)$, the weights $w(q)$, and the Pareto optimal allocation $\alpha_{1}(q)$ defined by (4.4)-(4.6). Clearly, by the construction of the random field $\pi=\pi(a)$, the total endowment of $\alpha_{1}(q)$ equals $\Sigma(x(q), q)$. Denoting

$$
v(q) \triangleq \frac{\partial G_{0}}{\partial u^{m}}\left(U_{0}, 1, q\right) \quad \text { and } \quad \widetilde{a}(q) \triangleq(v(q), x(q), q),
$$

we deduce from the equivalence of items 1 and 2 above that

$$
\begin{aligned}
U_{0}^{m} & =\frac{\partial F_{0}}{\partial v^{m}}(\widetilde{a}(q)), \quad m=1, \ldots, M, \\
1 & =\frac{\partial F_{0}}{\partial x}(\widetilde{a}(q)),
\end{aligned}
$$

and then from (4.2) that

$$
\frac{\partial G_{0}}{\partial q^{j}}\left(U_{0}, 1, q\right)=-\frac{\partial F_{0}}{\partial q^{j}}(\widetilde{a}(q)), \quad j=1, \ldots, J .
$$


Accounting for (4.1) and the constructions of $\pi(a)$ and $\mathbb{Q}(a)$ in (3.8) and (3.9) we obtain

$$
\begin{aligned}
U_{0}^{m} & =\mathbb{E}\left[\frac{\partial r}{\partial v^{m}}(v(q), \Sigma(x(q), q))\right]=\mathbb{E}\left[u_{m}\left(\pi^{m}(\widetilde{a}(q))\right],\right. \\
1 & =\mathbb{E}\left[\frac{\partial r}{\partial x}(v(q), \Sigma(x(q), q))\right], \\
\frac{\partial G_{0}}{\partial q^{j}}\left(U_{0}, 1, q\right) & =-\mathbb{E}\left[\frac{\partial r}{\partial x}(v(q), \Sigma(x(q), q)) \psi^{j}\right]=-\mathbb{E}_{\mathbb{Q}(\widetilde{a}(q))}\left[\psi^{j}\right] .
\end{aligned}
$$

As $w(q)$ is a scalar multiple of $v(q)$, we have $\pi(a(q))=\pi(\widetilde{a}(q))$ and $\mathbb{Q}_{a(q)}=$ $\mathbb{Q}_{\widetilde{a}(q)}$ and the equalities (4.3) and (4.7) follow.

Finally, the convexity and the continuous differentiability of $x=x(q)$ follow from (4.6) and the properties of $G_{0}$ given in Theorem 4.1 in [6].

Hereafter, we denote by $x=x(q): \mathbf{R}^{J} \rightarrow \mathbf{R}$ and $w=w(q): \mathbf{R}^{J} \rightarrow$ $\mathbf{S}^{M}$ the market indifference price and the weights of the post-trade Pareto allocation defined by (4.6) and (4.5), respectively. The sensitivity analysis of these maps is accomplished in Theorem 4.2 below, whose formulation requires us to introduce extra notations.

From now on, we also work under Assumption 2.2. We define the market makers' terminal risk tolerances

$$
\tau^{m}(a) \triangleq \frac{1}{a_{m}\left(\pi^{m}(a)\right)}=-\frac{u_{m}^{\prime}\left(\pi^{m}(a)\right)}{u_{m}^{\prime \prime}\left(\pi^{m}(a)\right)}, \quad m=1, \ldots, M, a \in \mathbf{A},
$$

and the aggregate terminal risk-tolerance

$$
R_{1}(a) \triangleq \sum_{m=1}^{M} \tau^{m}(a), \quad a=(v, x, q) \in \mathbf{A} .
$$

Taking constant $c>0$ from Assumption 2.2 we have

$$
\frac{1}{c} \leq \tau^{m}(a) \leq c, \quad m=1, \ldots, M, a \in \mathbf{A},
$$

and then

$$
\frac{M}{c} \leq R_{1}(a) \leq M c, \quad a \in \mathbf{A}
$$

The latter estimate allows us to define a probability measure $\mathbb{R}(a)$ whose density under $\mathbb{Q}(a)$ is given by

$$
\frac{d \mathbb{R}(a)}{d \mathbb{Q}(a)} \triangleq \frac{1 / R_{1}(a)}{\mathbb{E}_{\mathbb{Q}(a)}\left[1 / R_{1}(a)\right]}, \quad a \in \mathbf{A},
$$


as well as the initial aggregate risk-tolerance:

$$
R_{0}(a) \triangleq \mathbb{E}_{\mathbb{R}(a)}\left[R_{1}(a)\right], \quad a \in \mathbf{A}
$$

The terminology is due to the fact that $R=\left(R_{0}, R_{1}\right)$ is the stochastic field of risk-tolerances of the stochastic field $F=\left(F_{0}, F_{1}\right)$ of aggregate utilities:

$$
R_{i}(a)=-\frac{\partial F_{i}}{\partial x}(a) / \frac{\partial^{2} F_{i}}{\partial x^{2}}(a), \quad i=1,2 ;
$$

see the discussion in [6] just before Lemma 4.8 .

Finally, for $a \in \mathbf{A}$, we define the matrices

$$
\begin{aligned}
A^{l m}(a) & =\frac{1}{R_{0}(a)}\left\{\delta_{l m} \mathbb{E}_{\mathbb{R}(a)}\left[\tau^{m}(a) R_{1}(a)\right]-\operatorname{Cov}_{\mathbb{R}(a)}\left[\tau^{l}(a), \tau^{m}(a)\right]\right\}, \\
C^{m j}(a) & =\frac{1}{R_{0}(a)} \operatorname{Cov}_{\mathbb{R}(a)}\left[\tau^{m}(a), \psi^{j}\right], \\
D^{i j}(a) & =\frac{1}{R_{0}(a)} \operatorname{Cov}_{\mathbb{R}(a)}\left[\psi^{i}, \psi^{j}\right],
\end{aligned}
$$

where $\delta_{l m}=1_{\{l=m\}}$ is Kronecker's delta, we let $l, m=1, \ldots, M$ and $i, j=$ $1, \ldots, J$, and where

$$
\operatorname{Cov}_{\mathbb{R}}[\xi, \eta] \triangleq \mathbb{E}_{\mathbb{R}}[\xi \eta]-\mathbb{E}_{\mathbb{R}}[\xi] \mathbb{E}_{\mathbb{R}}[\eta]
$$

denotes the covariance of random variables $\xi$ and $\eta$ under a probability measure $\mathbb{R}$. Lemma 4.8 in [6] shows that $A(a)$ is strictly positive definite:

$$
\frac{1}{c}|z|^{2} \leq\langle z, A(a) z\rangle \leq c|z|^{2}, \quad z \in \mathbf{R}^{M},
$$

where $c>0$ is the constant from Assumption 2.2. In particular, the inverse matrix $A(a)^{-1}$ is well-defined.

Theorem 4.2. Let Assumptions 2.1, 2.2, and 2.4 hold. Then the Pareto weights $w=w(q)$ are continuously differentiable, the market indifference price $x=x(q)$ is two-times continuously differentiable and, for $m=1, \ldots, M$, $i, j=1, \ldots, J$, and $q \in \mathbf{R}^{J}$,

$$
\begin{aligned}
Z^{m j}(q) & \triangleq \frac{1}{w^{m}} \frac{\partial w^{m}}{\partial q^{j}}(q)=E^{m j}(q)-\sum_{k=1}^{M} w^{k}(q) E^{k j}(q), \\
\frac{\partial^{2} x}{\partial q^{i} \partial q^{j}}(q) & =H^{i j}(q)
\end{aligned}
$$


where the matrices $E=E(q)$ and $H=H(q)$ have the expressions

$$
\begin{aligned}
& E=-A^{-1} C, \\
& H=C^{T} A^{-1} C+D,
\end{aligned}
$$

in terms of the matrices $A \triangleq A(a(q)), C \triangleq C(a(q))$, and $D \triangleq D(a(q))$ computed in (4.10), (4.11), and (4.12) at $a(q) \triangleq(w(q), x(q), q)$.

The proof relies on the expressions below for the matrices $A(a), C(a)$, and $D(a)$ in terms of the partial derivatives of $F_{0}$. In the case of the matrix $A(a)$, such a formula is given in Lemma 4.8 of [6]:

$$
A^{l m}(v, x, q)=\frac{v^{l} v^{m}}{\frac{\partial F_{0}}{\partial x}}\left(\frac{\partial^{2} F_{0}}{\partial v^{l} \partial v^{m}}-\frac{1}{\frac{\partial^{2} F_{0}}{\partial x^{2}}} \frac{\partial^{2} F_{0}}{\partial v^{l} \partial x} \frac{\partial^{2} F_{0}}{\partial v^{m} \partial x}\right)(v, x, q) .
$$

The corresponding computations for the matrices $C(a)$ and $D(a)$ are done in the following lemma.

Lemma 4.3. Under Assumptions 2.1, 2.2, and 2.4, the matrices $C(a)$ and $D(a)$ defined by (4.11) and (4.12) can be written as

$$
\begin{aligned}
C^{m j}(v, x, q) & =\frac{v^{m}}{\frac{\partial F_{0}}{\partial x}}\left(\frac{\partial^{2} F_{0}}{\partial v^{m} \partial q^{j}}-\frac{1}{\frac{\partial^{2} F_{0}}{\partial x^{2}}} \frac{\partial^{2} F_{0}}{\partial v^{m} \partial x} \frac{\partial^{2} F_{0}}{\partial x \partial q^{j}}\right)(v, x, q), \\
D^{i j}(v, x, q) & =\frac{1}{\frac{\partial F_{0}}{\partial x}}\left(-\frac{\partial^{2} F_{0}}{\partial q^{i} \partial q^{j}}+\frac{1}{\frac{\partial^{2} F_{0}}{\partial x^{2}}} \frac{\partial^{2} F_{0}}{\partial x \partial q^{i}} \frac{\partial^{2} F_{0}}{\partial x \partial q^{j}}\right)(v, x, q) .
\end{aligned}
$$

Proof. From the identities for the second derivatives of $r=r(v, x)$ collected in Theorem 3.2 of [6] we deduce the following expressions for second order derivatives of $F_{1}(a)=r(v, \Sigma(x, q))$ :

$$
\begin{aligned}
v^{m} \frac{\partial^{2} F_{1}}{\partial v^{m} \partial x}(a) & =-\frac{\partial^{2} F_{1}}{\partial x^{2}}(a) \tau^{m}(a), \\
v^{m} \frac{\partial^{2} F_{1}}{\partial v^{m} \partial q^{j}}(a) & =-\frac{\partial^{2} F_{1}}{\partial x^{2}}(a) \tau^{m}(a) \psi^{j}, \\
\frac{\partial^{2} F_{1}}{\partial x \partial q^{j}}(a) & =\frac{\partial^{2} F_{1}}{\partial x^{2}}(a) \psi^{j}, \\
\frac{\partial^{2} F_{1}}{\partial q^{i} \partial q^{j}}(a) & =\frac{\partial^{2} F_{1}}{\partial x^{2}}(a) \psi^{i} \psi^{j} .
\end{aligned}
$$


By Theorem 4.2 in [6], the function $F_{0}=F_{0}(a)$ is twice continuously differentiable and its second order derivatives are expectations of the corresponding derivatives for $F_{1}=F_{1}(a)$ :

$$
\frac{\partial^{2} F_{0}}{\partial a^{i} \partial a^{j}}(a)=\mathbb{E}\left[\frac{\partial^{2} F_{1}}{\partial a^{i} \partial a^{j}}(a)\right] .
$$

The result now follows by direct computations if we account for the identities (4.9) for $R_{i}(a), i=1,2$ and the expression

$$
\frac{d \mathbb{R}(a)}{d \mathbb{P}} \triangleq \frac{\partial^{2} F_{1}}{\partial x^{2}}(a) / \frac{\partial^{2} F_{0}}{\partial x^{2}}(a),
$$

for the density of $\mathbb{R}(a)$ under $\mathbb{P}$.

Proof of Theorem 4.2. Theorem 4.2 in [6] implies that $G_{0}=G_{0}(a)$ is twice continuously differentiable. In view of the representations (4.5), for $w(q)$, and (4.6), for $x(q)$, we obtain that $w=w(q)$ is continuously differentiable and $x=x(q)$ is twice continuously differentiable. We also deduce that the identities (4.13) and (4.14) hold with the matrices $E(q)$ and $H(q)$ such that

$$
\begin{aligned}
E^{m j}(q) & =\frac{1}{\frac{\partial G_{0}}{\partial u^{m}}\left(U_{0}, 1, q\right)} \frac{\partial^{2} G_{0}}{\partial u^{m} \partial q^{j}}\left(U_{0}, 1, q\right), \\
H^{i j}(q) & =\frac{\partial^{2} G_{0}}{\partial q^{i} \partial q^{j}}\left(U_{0}, 1, q\right),
\end{aligned}
$$

where $m=1, \ldots, M$ and $i, j=1, \ldots, J$. The fact that these matrices can be computed by (4.15) and (4.16) is proved in Theorems 4.2 and 2.10 of [6] in the situation where the matrices $A(a), C(a)$, and $D(a)$ have the above expressions in terms of $F_{0}$.

\subsection{Asymptotic expansions for market indifference prices}

To facilitate the economic interpretation of the sensitivities given in Theorem 4.1 let us compute the second-order expansion for the market indifference prices $x=x(q)$ with respect to the order size $q$. Some additional notations are needed. For a random variable $\xi$ and a probability measure $\mathbb{R}$ denote

$$
\operatorname{Var}_{\mathbb{R}}[\xi] \triangleq \operatorname{Cov}_{\mathbb{R}}[\xi, \xi],
$$


the variance of $\xi$ under $\mathbb{R}$. For vectors $\mu \in \mathbf{S}^{M}$ and $z \in \mathbf{R}^{M}$ we shall use a similar notation:

$$
\operatorname{Var}_{\mu}[z] \triangleq \sum_{i=1}^{M} \mu^{i}\left(z^{i}\right)^{2}-\left(\sum_{i=1}^{M} \mu^{i} z^{i}\right)^{2}
$$

interpreted as the variance of the random variable $z$ defined on the sample space $\{1, \ldots, M\}$ with respect to the probability measure $\mu$. For $a \in \mathbf{A}$ define $\rho(a) \in \mathbf{L}^{0}\left(\mathbf{S}^{M}\right)$, the vector of relative risk-tolerance weights, by

$$
\rho^{m}(a) \triangleq \frac{\tau^{m}(a)}{\sum_{k=1}^{M} \tau^{k}(a)}=\frac{\tau^{m}(a)}{R_{1}(a)}, \quad m=1, \ldots, M .
$$

Theorem 4.4. Under Assumptions 2.1, 2.2, and 2.4, the second-order expansion for the market indifference prices $x=x(q)$ with respect to the order $q \in \mathbf{R}^{J}$ can be written as

$$
\begin{aligned}
x(q+\Delta q)-x(q) & =-\mathbb{E}_{\mathbb{Q}}[\langle\Delta q, \psi\rangle]+\frac{R_{0}}{2} \mathbb{E}_{\mathbb{R}}\left[\left(\frac{d \mathbb{Q}}{d \mathbb{R}}\right)^{2} \operatorname{Var}_{\rho}[Z \Delta q]\right] \\
& +\frac{1}{2 R_{0}}\left\{\left(\operatorname{Cov}_{\mathbb{R}}\left[\frac{d \mathbb{Q}}{d \mathbb{R}},\langle\Delta q, \psi\rangle\right]\right)^{2}+\operatorname{Var}_{\mathbb{R}}[\langle\Delta q, \psi\rangle]\right\} \\
& +o\left(|\Delta q|^{2}\right), \quad \Delta q \rightarrow 0,
\end{aligned}
$$

where $Z=Z(q)$ is defined in (4.13) and where we omitted the argument $a(q)=(w(q), x(q), q)$.

Proof. The linear term in (4.18) follows from (4.7). To verify the secondorder part we decompose the matrix $A=A(a(q))$ from (4.10) as

$$
A=R_{0}\left(S+s s^{T}\right)
$$

where, for $l, m=1, \ldots, M$,

$$
\begin{aligned}
S^{l m} & \triangleq \frac{1}{R_{0}^{2}} \mathbb{E}_{\mathbb{R}}\left[\tau^{l}\left(\delta_{l m} \sum_{k=1}^{M} \tau^{k}-\tau^{m}\right)\right], \\
s^{l} & \triangleq \frac{1}{R_{0}} \mathbb{E}_{\mathbb{R}}\left[\tau^{l}\right] .
\end{aligned}
$$


Denote $\mathbf{1} \triangleq(1, \ldots, 1) \in \mathbf{R}^{M}$ and observe that

$$
\begin{aligned}
S \mathbf{1} & =\left(\mathbf{1}^{T} S\right)^{T}=0, \\
\langle s, \mathbf{1}\rangle & =\frac{1}{R_{0}} \mathbb{E}_{\mathbb{R}}\left[\sum_{k=1}^{M} \tau^{k}\right]=\frac{1}{R_{0}} \mathbb{E}_{\mathbb{R}}\left[R_{1}\right]=1 .
\end{aligned}
$$

Let $E=E(q)$ and $H=H(q)$ be the matrices defined by (4.15) and (4.16). Using the fact that $A=A^{T}$ we can write $H$ as

$$
H=E^{T} A E+D .
$$

By Theorem 4.2, $H=H(q)$ is the Hessian matrix for $x=x(q)$. Hence, the second-order part in the expansion of $x(q+\Delta q)-x(q)$ with respect to $\Delta q$ is given by

$$
\begin{aligned}
\frac{1}{2}\langle\Delta q, H \Delta q\rangle & =\frac{1}{2}\langle E \Delta q, A E \Delta q\rangle+\frac{1}{2}\langle\Delta q, D \Delta q\rangle \\
& =\frac{R_{0}}{2}\langle E \Delta q, S E \Delta q\rangle+\frac{R_{0}}{2}(\langle E \Delta q, s\rangle)^{2}+\frac{1}{2}\langle\Delta q, D \Delta q\rangle .
\end{aligned}
$$

Since, by (4.13), $(E-Z) \Delta q$ is the product of some scalar on the vector 1, (4.19) implies that $\langle E \Delta q, S E \Delta q\rangle=\langle Z \Delta q, S Z \Delta q\rangle$. Observe that, in view of (4.8), the matrix $S$ can be written as

$$
S^{l m}=\frac{1}{R_{0}^{2}} \mathbb{E}_{\mathbb{R}}\left[R_{1}^{2} \rho^{l}\left(\delta_{l m}-\rho^{m}\right)\right]=\mathbb{E}_{\mathbb{R}}\left[\left(\frac{d \mathbb{Q}}{d \mathbb{R}}\right)^{2} \rho^{l}\left(\delta_{l m}-\rho^{m}\right)\right] .
$$

It follows that

$$
\langle Z \Delta q, S Z \Delta q\rangle=\mathbb{E}_{\mathbb{R}}\left[\left(\frac{d \mathbb{Q}}{d \mathbb{R}}\right)^{2} \operatorname{Var}_{\rho}[Z \Delta q]\right],
$$

giving the first quadratic term in (4.18).

By (4.15), $\langle\mathbf{1},(A E+C) \Delta q\rangle=0$, which, in view of (4.19) and (4.20), implies that

$$
R_{0}\langle s, E \Delta q\rangle+\langle\mathbf{1}, C \Delta q\rangle=0 .
$$

From the construction of $C$ in (4.11) and accounting again for (4.8) we deduce the second quadratic term in (4.18):

$$
\begin{aligned}
\langle s, E \Delta q\rangle & =-\frac{1}{R_{0}}\langle\mathbf{1}, C \Delta q\rangle=-\frac{1}{R_{0}^{2}} \operatorname{Cov}_{\mathbb{R}}\left[R_{1},\langle\Delta q, \psi\rangle\right] \\
& =-\frac{1}{R_{0}} \operatorname{Cov}_{\mathbb{R}}\left[\frac{d \mathbb{Q}}{d \mathbb{R}},\langle\Delta q, \psi\rangle\right] .
\end{aligned}
$$


Finally, the expression (4.12) for $D$ yields the last term:

$$
\langle\Delta q, D \Delta q\rangle=\frac{1}{R_{0}} \operatorname{Var}_{\mathbb{R}}[\langle\Delta q, \psi\rangle] .
$$

Observe that the linear term in (4.18) corresponds to the "standard" model of mathematical finance, where a "small" investor can trade any number of securities $\psi$ at "fixed" or exogenous prices $\mathbb{E}_{\mathbb{Q}}[\psi]$. The second, quadratic, component can thus be viewed as a price impact correction to this model. Note that all three terms of the quadratic part are non-negative and the last term, $\operatorname{Var}_{\mathbb{R}}[\langle\Delta q, \psi\rangle]$, equals zero iff $\langle\Delta q, \psi\rangle=$ const. Hence, for any non-trivial transaction our large investor will have to pay a strictly positive penalty due to his price impact in comparison with a hypothetical small agent trading at $\mathbb{E}_{\mathbb{Q}}[\psi]$.

To get a further insight into the microstructure of our model we provide still another second-order expansion of $x=x(q)$ along with the first-order expansion of the post-trade Pareto allocations $\pi=\pi(q) \triangleq \pi(a(q))$. We introduce probability measures $\mathbb{R}^{m}(a), m=1, \ldots, M$, such that

$$
\frac{d \mathbb{R}^{m}(a)}{d \mathbb{Q}(a)} \triangleq \frac{1 / \tau^{m}(a)}{\mathbb{E}_{\mathbb{Q}(a)}\left[1 / \tau^{m}(a)\right]}, \quad m=1, \ldots, M, a \in \mathbf{A} ;
$$

they are analogs of $\mathbb{R}(a)$ for individual market makers. We shall also make use of the random matrix $\zeta=\zeta(a)$ given, for $a \in \mathbf{A}$, by

$$
\zeta^{m j}(a) \triangleq R_{1}(a) \rho^{m}(a)\left(E^{m j}(a)-\sum_{l=1}^{M} \rho^{l}(a) E^{l j}(a)\right),
$$

where $m=1, \ldots, M, j=1, \ldots, J$, and the matrix $E=E(a)$ is defined in (4.15). We denote by $\zeta^{m}$ the $m$ th row of $\zeta$ and observe that

$$
\sum_{m=1}^{M} \zeta^{m}=0
$$

Theorem 4.5. Under Assumptions 2.1, 2.2, and 2.4, the first-order expansion, in the almost sure sense, of the Pareto optimal allocations $\pi=\pi(q) \triangleq$ $\pi(a(q))$ is given by

$$
\begin{aligned}
\pi^{m}(q+\Delta q)-\pi(q)= & \rho^{m}\left(\langle\psi, \Delta q\rangle-\mathbb{E}_{\mathbb{Q}}[\langle\psi, \Delta q\rangle]\right)+\left\langle\zeta^{m}, \Delta q\right\rangle \\
& +o\left(|\Delta q|^{2}\right), \quad \Delta q \rightarrow 0, m=1, \ldots, M,
\end{aligned}
$$


and the second-order expansion for the market indifference prices $x=x(q)$ can be written as

$$
\begin{aligned}
x(q+\Delta q)-x(q)= & -\mathbb{E}_{\mathbb{Q}}[\langle\Delta q, \psi\rangle] \\
& +\frac{1}{2}\left\{\mathbb{E}_{\mathbb{Q}}\left[1 / R_{1}\right] \mathbb{E}_{\mathbb{R}}\left[\left\langle\psi-\mathbb{E}_{\mathbb{Q}}[\psi], \Delta q\right\rangle^{2}\right]\right. \\
& \left.\quad+\sum_{m=1}^{M} \mathbb{E}_{\mathbb{Q}}\left[1 / \tau^{m}\right] \mathbb{E}_{\mathbb{R}^{m}}\left[\left\langle\zeta^{m}, \Delta q\right\rangle^{2}\right]\right\} \\
& +o\left(|\Delta q|^{2}\right), \quad \Delta q \rightarrow 0,
\end{aligned}
$$

where we omitted the argument $a(q)=(w(q), x(q), q)$.

Proof. To verify (4.23) recall that if $\widehat{x}^{m}=\widehat{x}^{m}(v, x), m=1, \ldots, M$, is the argmax vector in the construction of the aggregate utility:

$$
r(v, x) \triangleq \sup _{x^{1}+\cdots+x^{M}=x} \sum_{m=1}^{M} v^{m} u_{m}\left(x^{m}\right)=\sum_{m=1}^{M} v^{m} u_{m}\left(\widehat{x}^{m}\right),
$$

then its partial derivatives are given by

$$
\begin{aligned}
\frac{\partial \widehat{x}^{m}}{\partial x}(v, x) & =\frac{t_{m}\left(\widehat{x}^{m}\right)}{\sum_{k=1}^{M} t_{k}\left(\widehat{x}^{k}\right)}, \\
v^{l} \frac{\partial \widehat{x}^{m}}{\partial v^{l}}(v, x) & =v^{m} \frac{\partial \widehat{x}^{l}}{\partial v^{m}}(v, x)=t_{m}\left(\widehat{x}^{m}\right)\left(\delta_{l m}-\frac{t_{l}\left(\widehat{x}^{l}\right)}{\sum_{k=1}^{M} t_{k}\left(\widehat{x}^{k}\right)}\right),
\end{aligned}
$$

see, e.g., Theorem 3.2 in [6], where

$$
t_{m}(x) \triangleq \frac{1}{a_{m}(x)}=-\frac{u_{m}^{\prime}(x)}{u_{m}^{\prime \prime}(x)}, \quad x \in \mathbf{R}, m=1, \ldots, M,
$$

are the market makers' risk-tolerances. It follows that, for $a=(v, x, q)$,

$$
\begin{aligned}
\frac{\partial \pi^{m}}{\partial x}(a) & =\rho^{m}(a), \\
\frac{\partial \pi^{m}}{\partial q^{j}}(a) & =\rho^{m}(a) \psi^{j}, \\
v^{l} \frac{\partial \pi^{m}}{\partial v^{l}}(a) & =R_{1}(a) \rho^{m}(a)\left(\delta_{l m}-\rho^{l}(a)\right) .
\end{aligned}
$$


Combining these identities with (4.7) and (4.13) and omitting, as usual, the argument $a(q)=(w(q), x(q), q)$ we obtain

$$
\begin{aligned}
\frac{\partial}{\partial q^{j}} \pi^{m}(q)= & \rho^{m} \frac{\partial x(q)}{\partial q^{j}}+\rho^{m} \psi^{j}+R_{1}(a) \rho^{m}(a) \sum_{l=1}^{M}\left(\delta_{l m}-\rho^{l}(a)\right) \frac{1}{w^{l}(q)} \frac{\partial w^{l}(q)}{\partial q^{j}} \\
= & \rho^{m}\left(-\mathbb{E}_{\mathbb{Q}}\left[\psi^{j}\right]\right)+\rho^{m} \psi^{j} \\
& +R_{1}(a) \rho^{m}(a) \sum_{l=1}^{M}\left(\delta_{l m}-\rho^{l}(a)\right)\left(E^{l j}(q)-\sum_{k=1}^{M} w^{k}(q) E^{k j}(q)\right) \\
= & \rho^{m}\left(\psi^{j}-\mathbb{E}_{\mathbb{Q}}\left[\psi^{j}\right]\right)+\zeta^{m j},
\end{aligned}
$$

which yields (4.23).

To verify the second-order expansion (4.24) for $x=x(q)$ we shall match its terms with those of (4.18). Recall that $d \mathbb{Q} / d \mathbb{R}=R_{1} / R_{0}$ and observe that for a random variable $\xi$

$$
\begin{aligned}
\mathbb{E}_{\mathbb{R}}\left[\left(\xi-\mathbb{E}_{\mathbb{Q}}[\xi]\right)^{2}\right. & =\mathbb{E}_{\mathbb{R}}\left[\left(\xi-\mathbb{E}_{\mathbb{R}}[\xi]\right)^{2}+\left(\mathbb{E}_{\mathbb{R}}[\xi]-\mathbb{E}_{\mathbb{Q}}[\xi]\right)^{2}\right. \\
& =\operatorname{Var}_{\mathbb{R}}[\xi]+\left(\operatorname{Cov}_{\mathbb{R}}\left[\frac{d \mathbb{Q}}{d \mathbb{R}}, \xi\right]\right)^{2} .
\end{aligned}
$$

This shows that the second term in (4.24) coincides with the sum of the last two terms in (4.18).

If now $\eta=\left(\eta^{m}\right)_{m=1, \ldots, M}$ is a vector of random variables such that $\langle\rho, \eta\rangle=$ $\sum_{m=1}^{M} \rho^{m} \eta^{m}=0$, then

$$
\begin{aligned}
& \sum_{m=1}^{M} \mathbb{E}_{\mathbb{Q}}\left[1 / \tau^{m}\right] \mathbb{E}_{\mathbb{R}^{m}}\left[\left(\tau^{m} \eta^{m}\right)^{2}\right]=\sum_{m=1}^{M} \mathbb{E}_{\mathbb{Q}}\left[\tau^{m}\left(\eta^{m}\right)^{2}\right] \\
& =R_{0} \sum_{m=1}^{M} \mathbb{E}_{\mathbb{R}}\left[\left(\frac{d \mathbb{Q}}{d \mathbb{R}}\right)^{2} \rho^{m}\left(\eta^{m}\right)^{2}\right]=R_{0} \mathbb{E}_{\mathbb{R}}\left[\left(\frac{d \mathbb{Q}}{d \mathbb{R}}\right)^{2} \operatorname{Var}_{\rho}[\eta]\right] .
\end{aligned}
$$

Choosing

$$
\eta^{m}=\frac{1}{\tau^{m}}\left\langle\zeta^{m}, \Delta q\right\rangle, \quad m=1, \ldots, M,
$$

we deduce from (4.22) that $\langle\rho, \eta\rangle=0$ and then from (4.21) and (4.13) that

$$
\eta=E \Delta q+z_{1} \mathbf{1}=Z \Delta q+z_{2} \mathbf{1}
$$


where $\mathbf{1}=\{1, \ldots, 1\}$ and $z_{1}$ and $z_{2}$ are some constants. It follows that

$$
\operatorname{Var}_{\rho}[\eta]=\operatorname{Var}_{\rho}[E \Delta q]=\operatorname{Var}_{\rho}[Z \Delta q]
$$

proving the equality between the third term in (4.24) and the second term in (4.18).

The expansion (4.23) shows that the market makers share the extra endowment $\left\langle\psi-\mathbb{E}_{\mathbb{Q}}[\psi], \Delta q\right\rangle$ in proportion to their individual risk tolerances and, in addition, make zero-sum trades $\left\langle\zeta^{m}, \Delta q\right\rangle, m=1, \ldots, M$, between themselves; see (4.22). As the expansion (4.24) reveals, the liquidity premium arises from both these components.

It is quite common in the economic literature to replace a collection of economic agents with a single, representative, agent whose utility function is given by $r(w, \cdot)$, with $r=r(v, x)$ from (3.1), for some fixed weight $w \in \mathbf{S}^{M}$. In our case, this simplification yields the linear term in (4.24) as well as the first component of the quadratic part, but not the remaining ones. It is interesting to obtain conditions for the second term of the quadratic part in (4.24) or, equivalently, the second term in (4.18) to vanish, since, then, the representative agent approximation leads to the identical expression for the price impact coefficient as our original model with many market makers. This is accomplished in the following lemma.

Lemma 4.6. Let the conditions of Theorem 4.4 hold and take $q, \Delta q \in \mathbf{R}^{J}$. Then the following assertions are equivalent:

1. $\mathbb{E}_{\mathbb{R}^{m}}\left[\left\langle\zeta^{m}, \Delta q\right\rangle^{2}\right]=0, m=1, \ldots, M$.

2. $\mathbb{E}_{\mathbb{R}}\left[\left(\frac{d \mathbb{Q}}{d \mathbb{R}}\right)^{2} \operatorname{Var}_{\rho}[Z(q) \Delta q]\right]=0$;

3. $Z(q) \Delta q=0$;

4. $\mathbb{E}_{\mathbb{Q}^{m}}[\langle\Delta q, \psi\rangle]=\mathbb{E}_{\mathbb{Q}}[\langle\Delta q, \psi\rangle], m=1, \ldots, M$,

where, for $a \in \mathbf{A}$,

$$
\frac{d \mathbb{Q}^{m}(a)}{d \mathbb{Q}(a)} \triangleq \frac{\rho^{m}(a)}{\mathbb{E}_{\mathbb{Q}}\left[\rho^{m}(a)\right]}, \quad m=1, \ldots, M,
$$

and we omitted the argument $a(q) \triangleq(w(q), x(q), q)$. 
Proof. $1 \Longleftrightarrow 2$ : This follows from the equality:

$$
\sum_{m=1}^{M} \mathbb{E}_{\mathbb{Q}}\left[1 / \tau^{m}\right] \mathbb{E}_{\mathbb{R}^{m}}\left[\left\langle\zeta^{m}, \Delta q\right\rangle^{2}\right]=R_{0} \mathbb{E}_{\mathbb{R}}\left[\left(\frac{d \mathbb{Q}}{d \mathbb{R}}\right)^{2} \operatorname{Var}_{\rho}[Z \Delta q]\right]
$$

which was part of the proof of Theorem 4.5.

$2 \Longleftrightarrow 3$ : Clearly, item 2 holds if and only if $\operatorname{Var}_{\rho}[Z(q) \Delta q]=0$, which, in turn, is equivalent to $Z(q) \Delta q=y \mathbf{1}$ for some $y \in \mathbf{R}$. From the construction of the matrix $Z(q)$ in (4.13) we deduce that $\langle w(q), Z(q) \Delta q\rangle=0$, where the Pareto weights $w(q)$ take values in $\mathbf{S}^{M}$. It follows that $y=0$.

$3 \Longleftrightarrow 4$ : Denote $\xi \triangleq\langle\Delta q, \psi\rangle \in \mathbf{L}^{0}(\mathbf{R})$. From the definition of the measures $\mathbb{Q}^{m}, m=1, \ldots, M$, we deduce the equivalence of item 4 to

$$
\mathbb{E}_{\mathbb{Q}^{m}}[\xi]=\mathbb{E}_{\mathbb{Q}^{1}}[\xi], \quad m=2, \ldots, M .
$$

From (4.13) we deduce that $Z(q) \Delta q=0$ if and only if $E \Delta q=y \mathbf{1}$ for some $y \in$ $\mathbf{R}$, where the matrix $E=E(q)$ satisfies (4.15). Hence, item 3 is equivalent to the existence of a constant $y \in \mathbf{R}$ such that

$$
y A 1+C \Delta q=0,
$$

From the expressions (4.10) and (4.11) for the matrices $A=A(a(q))$ and $C=C(a(q))$ we obtain

$$
\begin{aligned}
(A \mathbf{1})^{m} & =\mathbb{E}_{\mathbb{R}}\left[\tau^{m}\right]=R_{0} \mathbb{E}_{\mathbb{Q}}\left[\rho^{m}\right], \\
(C \Delta q)^{m} & =\frac{1}{R_{0}} \operatorname{Cov}_{\mathbb{R}}\left[\tau^{m}, \xi\right]=\frac{1}{R_{0}}\left(\mathbb{E}_{\mathbb{R}}\left[\tau^{m} \xi\right]-\mathbb{E}_{\mathbb{R}}\left[\tau^{m}\right] \mathbb{E}_{\mathbb{R}}[\xi]\right) \\
& =\mathbb{E}_{\mathbb{Q}}\left[\rho^{m}\right]\left(\mathbb{E}_{\mathbb{Q}^{m}}[\xi]-\mathbb{E}_{\mathbb{R}}[\xi]\right), \quad m=1, \ldots, M,
\end{aligned}
$$

which clearly implies the equivalence of (4.25) and (4.26).

The condition of item 4 is clearly satisfied when the random weights $\rho$ defined in (4.17) are deterministic. This is the case, for instance, if all market makers have exponential utilities: $u_{m}(x)=-\exp \left(-a_{m} x\right)$, with constant riskaversion $a_{m}>0, m=1, \ldots, M$. Moreover, if the securities $\psi$ form a complete model in the sense that, jointly with the constant security paying 1 they span all random variables, than the validity of item 4 for any $\Delta q \in \mathbf{R}^{J}$ is in fact equivalent to $\rho$ being deterministic. 


\section{References}

[1] Robert Almgren and Neil Chriss. Optimal execution of portfolio transactions. J. Risk, 3:5-39, 2001.

[2] Yakov Amihud, Haim Mendelson, and Lasse H. Pedersen. Liquidity and asset prices. Foundations and Trends in Finance, 1(4):269-364, 2005.

[3] Robert M. Anderson and Roberto C. Raimondo. Equilibrium in continuous-time financial markets: endogenously dynamically complete markets. Econometrica, 76(4):841-907, 2008. ISSN 0012-9682.

[4] Kerry Back. Insider trading in continuous time. Review of Financial Studies, 5:387-409, 1992.

[5] Peter Bank and Dmitry Kramkov. A model for a large investor trading at market indifference prices. II: continuous-time case. arXiv:1110.3229v2, October 2011. URL http://arxiv.org/abs/1110.3229v2.

[6] Peter Bank and Dmitry Kramkov. The stochastic field of aggregate utilities and its saddle conjugate. arXiv:1310.7280, October 2013. URL http://arxiv.org/abs/1310.7280.

[7] Dan Bernhardt and Eric Hughson. Splitting orders. Review of Financial Studies, 10:69-101, 1997.

[8] Bruno Biais, David Martimort, and Jean-Charles Rochet. Competing mechanisms in a commun value environment. Econometrica, 68(4):799$837,2000$.

[9] Bruno Biais, Larry Glosten, and Chester Spatt. Market microstructure: A survey of microfoundations, empirical results, and policy implication. Journal of Financial Markets, 8:217-264, 2005.

[10] Umut Çetin, Robert A. Jarrow, and Philip Protter. Liquidity risk and arbitrage pricing theory. Finance Stoch., 8(3):311-341, 2004. ISSN 09492984.

[11] Jakša Cvitanić and Jin Ma. Hedging options for a large investor and forward-backward SDE's. Ann. Appl. Probab., 6(2):370-398, 1996. ISSN 1050-5164. 
[12] Rose Anne Dana and Cuong Le Van. Asset equilibria in $L^{p}$ spaces with complete markets: a duality approach. J. Math. Econom., 25(3):263280, 1996. ISSN 0304-4068. doi: 10.1016/0304-4068(95)00735-0. URL http://dx.doi.org/10.1016/0304-4068(95)00735-0.

[13] Freddy Delbaen and Walter Schachermayer. A general version of the fundamental theorem of asset pricing. Math. Ann., 300(3):463-520, 1994. ISSN 0025-5831.

[14] Darrell Duffie, Nicolae Garleanu, and Lasse H. Pedersen. Over-thecounter-markets. Econometrica, 73:1815-1847, 2005.

[15] Rüdiger Frey and Alexander Stremme. Market volatility and feedback effects from dynamic hedging. Math. Finance, 7(4):351-374, 1997. ISSN 0960-1627.

[16] Nicolae Garleanu, Lasse Heje Pedersen, and Allen M. Poteshman. Demand-based option pricing. Rev. Financ. Stud., 22(10):4259-4299, 2009. doi: 10.1093/rfs/hhp005.

[17] Lawrence R. Glosten and Paul R. Milgrom. Bid, ask and transaction prices in a specialist market with heterogeneously informed traders. Journal of Financial Economics, 14(1):71 - 100, 1985. ISSN 0304-405X. doi: 10.1016/0304-405X(85)90044-3.

[18] Selim Gökay, Alexandre F. Roch, and H. Mete Soner. Liquidity models in continuous and discrete time. In Giulia Di Nunno and Bernt Øksendal, editors, Advanced Mathematical Methods for Finance, pages 333-365. Springer Berlin Heidelberg, 2011. ISBN 978-3-642-18412-3. URL http://dx.doi.org/10.1007/978-3-642-18412-3_13.

[19] Sanford J. Grossman and Merton H. Miller. Liquidity and market structure. The Journal of Finance, 43(3):617-633, 1988. ISSN 0022-1082.

[20] Thomas S.Y. Ho and Hans R. Stoll. Optimal dealer pricing under transactions and return uncertainty. Jounral of Financial Economics, 9:47$73,1981$.

[21] J. Hugonnier, S. Malamud, and E. Trubowitz. Endogenous completeness of diffusion driven equilibrium markets. Econometrica, 80(3): 
1249-1270, 2012. ISSN 1468-0262. doi: 10.3982/ECTA8783. URL http://dx.doi.org/10.3982/ECTA8783.

[22] Dmitry Kramkov. Existence of endogenously complete equilibrium driven by diffusion. arxiv:1304.3516v1, April 2013. URL http://arxiv.org/abs/1304.3516v1.

[23] Albert S. Kyle. Continuous auctions and insider trading. Econometrica, 53:1315-1335, 1985.

[24] Francis A. Longstaff. Optimal portfolio choice and the valuation of illiquid securities. The Review of Financial Studies, 14(2):407-431, 2001.

[25] Maureen O'Hara. Market Microstructure Theory. Blackwell, 1995. ISBN 978-1-55786-443-7.

[26] George Papanicolaou and Ronnie Sircar. General Black-Scholes models accounting for increased market volatility from hedging strategies. Applied Mathematical Finance, 5:45-82, 1998.

[27] Eckhard Platen and Martin Schweizer. On feedback effects from hedging derivatives. Math. Finance, 8(1):67-84, 1998. ISSN 0960-1627.

[28] Frank Riedel and Frederik Herzberg. Existence of financial equilibria in continuous time with potentially complete markets. J. Math. Econom., 49(5):398-404, 2013. ISSN 0304-4068. doi: 10.1016/j.jmateco.2013.07. 001. URL http://dx.doi.org/10.1016/j . jmateco.2013.07.001.

[29] R. Tyrrell Rockafellar. Convex analysis. Princeton Mathematical Series, No. 28. Princeton University Press, Princeton, N.J., 1970.

[30] Alexander Schied and Torsten Schöneborn. Risk aversion and the dynamics of optimal liquidation strategies in illiquid markets. Finance Stoch., 13(2):181-204, 2009. ISSN 0949-2984.

[31] Hans R. Stoll. The supply of dealer services in securities markets. The Journal of Finance, 33(4):1133-1151, 1978. ISSN 00221082.

[32] Marek Weretka. Endogenous market power. Journal of Economic Theory, 146(6):2281-2306, 2011. 